\title{
ARTICLE
}

\section{Carbohydrate-deficient glycoprotein syndrome type 1A: expression and characterisation of wild type and mutant PMM2 in E. coli}

\author{
Susanne Kjaergaard, Flemming Skovby and Marianne Schwartz \\ Department of Clinical Genetics, Rigshospitalet, University Hospital of Copenhagen, Denmark
}

\begin{abstract}
We have identified the PMM2 genotypes of 22 unrelated Danish patients with carbohydratedeficient glycoprotein syndrome type 1A: R141H/F119L (18), R141H/C192G (1), F119L/F119L (1), F119L/G117R (1) and D223E/T237R (1). The lack of patients homozygous for R141H is statistically highly significant, but unexplained. In order to investigate the effect of PMM2 mutations on phosphomannomutase (PMM2) activity, PMM2-cDNA was cloned into a pET3a vector. Following introduction of mutations into PMM2-cDNA by site-specific mutagenesis, wild type and mutant PMM2-cDNA were expressed in $E$. coli B121(DE3) cells, and the activity of PMM2 was determined by an enzymatic assay using mannose 1-phosphate as substrate. Recombinant R141H, G117R, and T237R PMM2 had no detectable catalytic activity, and the F119L PMM2 had 25\% of the activity of the wild type. The activity of the C192G and D223E PMM2 was in the normal range, but the affinity for their substrate was lower, and the proteins were more sensitive to increased temperatures. Each patient has at least one mutation which retains residual PMM2 activity. Our results support the hypotheses that a genotype conveying residual PMM2 catalytic activity is required for survival, and that homozygosity for R141H impairs PMM2 to a degree incompatible with life.
\end{abstract}

Keywords: carbohydrate-deficient glycoprotein syndrome 1A; PMM2; phosphomannomutase; gene expression

\section{Introduction}

Carbohydrate-deficient glycoprotein syndrome type IA (CDG1A, OMIM 212065) is an autosomal recessive disorder characterised by defective protein glycosylation due to deficiency of phosphomannomutase (PMM). PMM converts mannose 6-phosphate to mannose 1-phosphate which is required for synthesis of

Correspondence: Susanne Kjaergaard MD, Department of Clinical Genetics, University Hospital, Rigshospitalet 4062, Blegdamsvej 9, DK-2100 Copenhagen, Denmark. Tel: + 45354545 92; Fax: + 4535454072 ; E-mail: susanne@rh.dk Received 5 July 1999; accepted 6 August 1999
GDP-mannose. Two types of human phosphomannomutase, PMM1 and PMM2, have been identified. ${ }^{1,2}$ Mutations in the PMM2 (OMIM 601785) gene located on chromosome $16 \mathrm{p} 13$ are responsible for CDG1A. ${ }^{2}$ Two independent surveys of patients of CDG1A found PMM2 mutations on 145 of 148 disease chromosomes $(98 \%))^{2-4}$ All but one of the 30 different mutations detected so far have been of the missense type. ${ }^{2-6}$ Whilst most patients are compound heterozygotes, homozygosity for the F119L mutation has been observed in a few instances, and one patient is known to be homozygous for D65Y., ${ }^{3,5,7}$ In contrast, patients homozygous for the R141H mutation, present in more than $75 \%$ of patients of Caucasian origin, have been 
conspicuously absent in results of the genotype analyses. $^{3,4}$ These observations suggest that homozygosity for $\mathrm{R} 141 \mathrm{H}$ may not be compatible with life. In order to investigate the effect of PMM2 mutations on PMM2 activity we have expressed wild type and mutant $P M M 2-c D N A$ in $E$. coli and compared specific activities, affinities for mannose 1-phosphate, and temperature stabilities of the corresponding proteins.

\section{Materials and Methods}

\section{Patients}

The clinical diagnosis of CDG1A in 28 patients from 22 unrelated families of Danish origin was substantiated by isoelectric focusing of transferrin, ${ }^{8,9}$ by documentation of PMM deficiency in cultured fibroblasts, ${ }^{10}$ and by genotype analysis. ${ }^{4}$ Subsequently, we have identified a novel PMM2 mutation, C192G, in one of our patients.

\section{Expression of Recombinant Wild Type and Mutant PMM2-cDNA}

Total RNA was prepared from cultured fibroblasts derived from a healthy control using RNeasy (Qiagen, Düsseldorf, Germany), cDNA was generated with GeneAmp RNAPCR kit (Perkin Elmer Applied Biosystems, Foster City, CA, USA) using MuLV reverse transcriptase and random hexamers as primers. The coding sequence of $P M M 2$ was amplified by $P f u$ DNA polymerase using the generated cDNA as a template. Primers introducing one Nde1 restriction site (5'-CGGCTAGAAACTGGGCATATGGCAGCGCCTGGCCCAGC-3') and one Bam H1 (5'-CTTGTCAGCCGGGATCCCGCCCCTCCCGCT-3') restriction site (underlined) were used. The PCR product (794 bp) was isolated by electrophoresis in a 1\% agarose gel, purified with the QiaQuick gel extraction kit (Qiagen) and digested with $N d e 1$ and Bam $\mathrm{H} 1$. The insert was then cloned into the Nde1 and Bam $\mathrm{H} 1$ digested expression vector pET3a $^{11}$ (Novagen, Madison, WI, USA) by the Rapid DNA Ligation Kit (Boehringer Mannheim, Mannheim, Germany). The pET3a-PMM2 construct was subsequently transformed into E. coli NovaBlue and purified using Wizard Plus SV Minipreps DNA Purification System (Promega, Madison, WI, USA). The mutations R141H, F119L, D223E, C192G, T237R and G117R were introduced into PMM2-cDNA by sitespecific mutagenesis, ${ }^{12}$ purified and cloned as mentioned above. All constructs (inserts) were sequenced in both directions to rule out any PCR errors and to confirm the introduction of mutations, using the BigDye Terminator Cycle sequencing Kit, ABI Prism 310 (Perkin Elmer Applied Biosystems). The purified plasmid constructs were used to transform E. coli B121 (DE3) cells, and the transformed cells were grown in LB medium containing $50 \mu \mathrm{g} / \mathrm{ml}$ ampicillin, at $37^{\circ} \mathrm{C}$, until $\mathrm{OD}_{600}$ reached 0.6 . Isopropylthiogalactoside (IPTG) was then added to a final concentration of $0.4 \mathrm{~mm}$, and incubation was continued for $3 \mathrm{~h}$. The cells were harvested by centrifugation and stored at $-80^{\circ} \mathrm{C}$ until further use.

\section{Phosphomannomutase Assay}

Cell extracts of $E$. coli were prepared by resuspending the cell pellet in a buffer containing $20 \mathrm{~mm}$ Hepes, $25 \mathrm{~mm} \mathrm{KCl,} 1 \mathrm{~mm}$ dithiothreitol, and leupeptin and antipain each at a concentration of $10 \mu \mathrm{g} / \mathrm{ml}$. The suspension was sonicated $2 \times 10 \mathrm{sec}-$ onds and centrifuged at $10000 \mathrm{~g}$ for $10 \mathrm{~min}$. The resulting extracts were used for assaying PMM by the method described by Van Schaftingen and Jaeken ${ }^{10}$ with the exception that glucose 1,6-diphosphate (Sigma, St. Louis, MO, USA) instead of mannose 1,6-diphosphate was used as cofactor. The rate of NADPH formation was followed for $5 \mathrm{~min}$. One unit equals the formation of $1 \mathrm{nmol} \mathrm{NADPH} / \mathrm{min}$.

$K_{m}$ Estimation The affinity for mannose 1-phosphate was determined for wild type, F119L, C192G and D223E PMM2. The rate of NADPH formation was followed for $5 \mathrm{~min}$ with increasing concentration of mannose 1-phosphate keeping constant the concentration of glucose 1,6-diphosphate.

Temperature Stability The cell extracts were incubated for $10 \mathrm{~min}$ at $37,39,41,43$ and $45^{\circ} \mathrm{C}$ and then kept on ice until determination of PMM activity.

Protein was determined using the method of BCA Protein assay (Pierce, Rockford, IL, USA) with bovine serum albumin as a standard.

\section{Results}

Table 1 shows the spectrum of Danish PMM2 mutations including one novel mutation in exon 7, 574T $\rightarrow \mathrm{G}$ (C192G). Except for one patient homozygous for F119L, all are compound heterozygotes.

Both wild type and mutant PMM2 were successfully produced in E. coli. After $3 \mathrm{~h}$ of IPTG induction more than $90 \%$ of the PMM2 protein was found in the soluble protein fraction, as determined by SDS-PAGE analysis (data not shown). All cDNA constructs seemed to give the same yield of PMM2 protein.

The specific activities of wild type and mutant PMM2 are shown in Table 2 . The catalytic activity of wild type PMM2 was $850 \mathrm{U} / \mathrm{mg}$, whilst induction of the vector without insert gave an activity in the range of 33-56 U/mg. The R141H, G117R and T237R PMM2 gave no detectable additional activity, ie, the activity

Table $1 \quad P M M 2$ mutations in 22 unrelated CDG1A patients of Danish origin

\begin{tabular}{ll}
\hline PMM2 genotype & No. of patients \\
\hline R141H/F119L & 18 \\
R141H/C192G & 1 \\
G117R/F119L & 1 \\
F119L/F119L & 1 \\
T237R/D223E & 1
\end{tabular}

The recombinant proteins corresponding to the mutations in bold have no detectable PMM2 activity 
Table 2 Specific activities of recombinant wild type and mutant PMM2

\begin{tabular}{lcl}
\hline Vector & $\begin{array}{l}\text { Specific activity } \\
\text { nmol/mean min/mg }\end{array}$ & Range \\
\hline pET3a & 45 & $(33-56)$ \\
pET3a-wtPMM2 & 849 & $(718-1046)$ \\
pET3a-F119L & 217 & $(143-289)$ \\
pET3a-R141H & 47 & $(31-63)$ \\
pET3a-G117R & 45 & $(29-61)$ \\
pET3a-D223E & 1022 & $(896-1152)$ \\
pET3a-T237R & 38 & $(25-50)$ \\
pET3a-C192G & 1101 & $(1009-1193)$ \\
\hline
\end{tabular}

was at the same level as if the vector had no insert. The activity of the F119L PMM 2 was $25 \%$ of that of the wild type. Surprisingly, the activities of the D223E and C192G PMM2 were found to be in the high normal range or above.

The D223E and C192G mutations were found in two patients with the genotype T237R/D223E and $\mathrm{R} 141 \mathrm{H} / \mathrm{C} 192 \mathrm{G}$, respectively. In order to exclude the presence of other mutations, cDNA from both alleles of the patients were cloned and sequenced. Each patient had the mutations in trans position, and no other mutation was detected in the coding sequences of $P M M 2$. Both cDNA alleles were of the correct size, and analysis of cDNA by agarose gel electrophoresis did not show any sign of alternative splicing. To characterise wild type and mutant proteins the apparent $\mathrm{K}_{\mathrm{m}}$ values for mannose 1-phosphate (Figure 1) as well as the temperature inactivation patterns were determined (Figure 2). The $\mathrm{K}_{\mathrm{m}}$ value of wild type PMM2 was $17 \mu \mathrm{M}$.

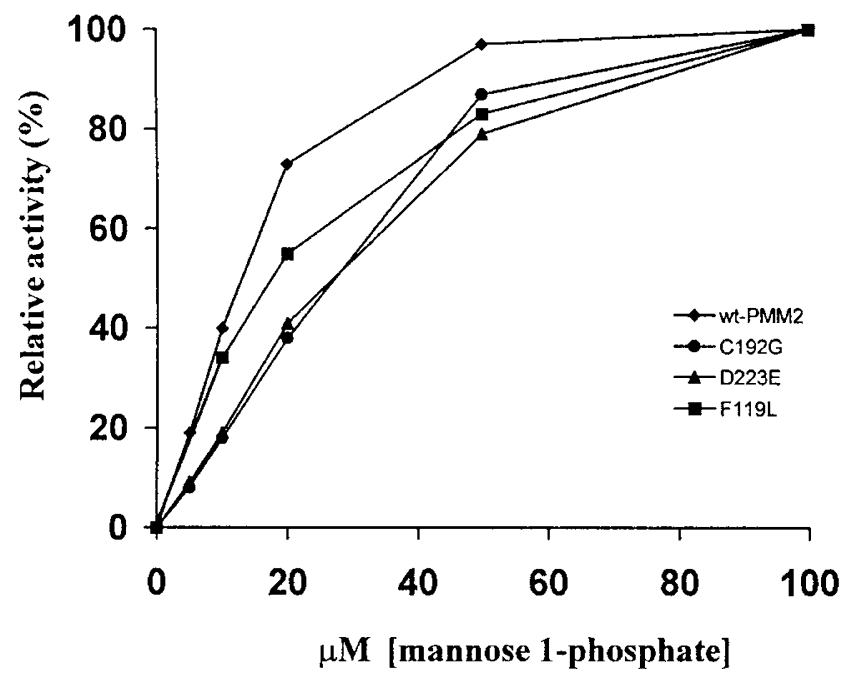

Figure 1 Saturation curves of recombinant wild type and mutant PMM2 with mannose 1-phosphate. The velocities are expressed as \% of the activity at $100 \mu \mathrm{M}$ mannose 1-phosphate

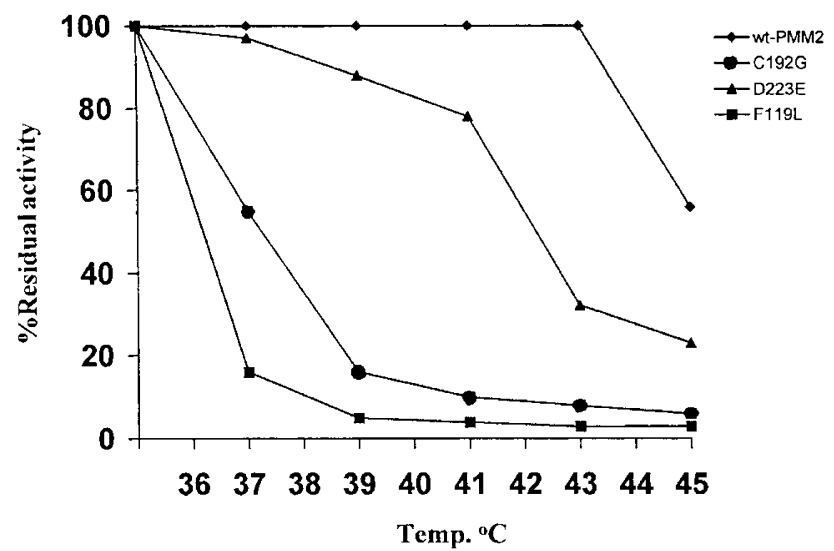

Figure 2 Temperature inactivation curves of recombinant wild type and mutant PMM2. The enzymatic activities are expressed as \% of the activity of the protein without temperature inactivation

The apparent $\mathrm{K}_{\mathrm{m}}$ of the F119L PMM2 was close to that of the wild type, whilst the G192C and D223E PMM2 had overlapping saturation curves indicating a lower affinity for mannose 1-phosphate.

Incubation of crude cell extracts at different temperatures (range $37-45^{\circ} \mathrm{C}$ ) showed that wild type PMM2 was relatively temperature resistant and that mutant proteins were inactivated rapidly by increasing temperatures. The temperature at which $50 \%$ activity remained was much lower for the $\mathrm{C} 192 \mathrm{G}$ and the F119L PMM2 than for the wild type, whilst the activity of the D223E PMM2 seemed more temperature resistant than that of the other mutant proteins.

\section{Discussion}

The six missense mutations identified in the Danish CDG1A patients result in replacement of amino acids which are conserved strictly among phosphomannomutases of yeast (SEC53), Candida albicans and human phosphomannomutases (PMM1 and PMM2), ${ }^{2,13}$ suggesting that these amino acids are crucial for the catalytic activity of the enzyme.

In order to explore the relationship between the mutant genes and their corresponding enzymes, we introduced all six mutations in PMM2-cDNA and expressed the recombinant proteins in E. coli. The specific activities measured suggest the presence of two types of mutation. One type results in proteins with zero activity and the other type results in proteins with decreased activity and/or altered characteristics such as 
decreased affinity for the substrate and less thermostability.

$\mathrm{R} 141 \mathrm{H}$ is the prevalent PMM2 mutation in the European population. ${ }^{3,4}$ So far R141H has not been observed in the homozygous form, although an incidence of approximately 1:20000 would be expected on the basis of the frequency of the R141H allele in the European population (E Schollen et al 1999, submitted). At present there is no basis for assuming that homozygosity for $\mathrm{R} 141 \mathrm{H}$ conveys a clinical phenotype different from classical CDG1A. F119L has been found in approximately $50 \%$ of Scandinavian patients, usually in the compound heterozygous form, but a few homozygous patients have been observed. ${ }^{4,5,7}$ So far, T237R, G117R, D223E and C192G have been seen only in compound heterozygous patients, and the latter three mutations only in Danish patients. ${ }^{3,4}$

Expression of the $\mathrm{R} 141 \mathrm{H}$ protein as well as the G117R and the T237R proteins showed repeatedly no measurable residual PMM2 activity. The mutant proteins were present in the soluble cellular fraction in quantities comparable to that of the wild type protein. When observed in patients, each of these three mutations was found in combination with a mutation with detectable PMM2 activity, F119L, C192G or D223E (Table1). Taken together, these results support the notion that lack of PMM2 activity is incompatible with life. The fate of the zygote with the R141/R141H genotype is unknown. Due to the fundamental importance of PMM2 in normal cellular functions, it is most likely that the zygote is lost in early pregnancy.

Surprisingly, expression of the C192G and the D223E PMM2 showed activities in the normal range or higher. Sequencing of the coding region of PMM2 of these alleles excluded the presence of other mutations which could account for the clinical phenotype. Subsequent studies of the affinity of the mutant proteins for mannose 1-phosphate and their temperature stability did in fact reveal altered properties of the recombinant C192G and D223E PMM2. Although we used crude E. coli extracts, our results suggest that the mutations interfere also in vivo with the properties of the PMM2 thereby accounting for the clinical phenotype of the patients.

Provided that mannose 1-phosphate is required for formation of GDP-mannose and that no alternative pathway exists for synthesis of mannose 1-phosphate, residual activity would be expected in mutant PMM2 since the majority of $\mathrm{N}$-linked carbohydrate chains is present on serum proteins of CDG1A patients. Thus far, alternative pathways to generate mannose 1-phosphate have not been described although the role of PMM1 is still a puzzle. Recent studies in the rat have shown that PMM2 appears to be practically the sole PMM present in most tissues. ${ }^{14}$ The lack of rescue by PMM1 in man might explain the multi-organ dysfunction of patients with CDG1A. Each of our patients has at least one mutation which retains PMM2 activity when expressed in E. coli (Table1). This residual activity, however, is not reflected in the specific activities found in cultured fibroblasts derived from the patients. ${ }^{4}$ Even the patient homozygous for F119L has undetectable PMM activity in extracts of cultured fibroblasts (data not shown). PMM of man is presumed to be a dimer, as in yeast $S E C 53,{ }^{15}$ and three different subunit combinations should therefore be present in compound heterozygous patients. The very low, or lack of, measurable PMM activity in fibroblasts could indicate a dominant-negative effect of the null activity polypeptides, resulting in a final catalytic activity less than expected from the genotype. However, due to the very low activity of PMM even in control fibroblasts, it is not possible to correlate the residual PMM activities with the genotypes.

Our results support the hypotheses that homozygosity for R141H impairs PMM2 activity to a degree incompatible with life, whilst F119L or other 'mild' mutations convey a phenotype compatible with survival due to residual PMM2 activity.

\section{Acknowledgements}

We are grateful to Dagmar Marshall's Foundation, King Christian IX and Queen Louise's Jubilee Foundation, John and Birthe Meyer's Foundation and the Novo Nordic Foundation for financial support.

Note: After submission of the manuscript we noticed a paper about the effect of mutations found in carbohydratedeficient glycoprotein syndrome type $1 \mathrm{~A}$ on the activity of the phosphomannomutase 2 (Pirard M, Matthijs G, Heykants L et al: FEBS Letters 1999; 452: 319-322). The results provided are in agreement with our findings.

\section{References}

1 Matthijs G, Schollen E, Pirard M et al: PMM (PMM1), the human homologue of SEC53 or yeast phosphomannomutase, is localized on chromosome 22q13. Genomics 1997; 40: $41-47$. 
2 Matthijs G, Schollen E, Pardon E et al: Mutations in $P M M 2$, a phosphomannomutase gene on chromosome 16p13, in carbohydrate-deficient glycoprotein type I syndrome (Jaeken syndrome). Nat Genet 1997; 16: 88-92.

3 Matthijs G, Schollen E, Van Schaftingen E, Cassiman JJ, Jaeken J: Lack of homozygotes for the most frequent disease allele in carbohydrate-deficient glycoprotein syndrome type 1A. Am J Hum Genet 1998; 62: 542-550.

4 Kjaergaard S, Skovby F, Schwartz M: Absence of homozygosity for predominant mutations in PMM2 in Danish patients with carbohydrate-deficient glycoprotein syndrome type 1. Eur J Hum Genet 1998; 6: 331-336.

5 Bjursell C, Wahlström J, Berg K et al: Detailed mapping of the phosphomannomutase 2 (PMM2) gene and mutation detection enable improved analysis for Scandinavian CDG type I families. Eur J Hum Genet 1998; 6: 603-611.

6 Kondo I, Mizugishi K, Yoneda Y et al: Missense mutations in phosphomannomutase 2 gene in two Japanese families with carbohydrate-deficient glycoprotein syndrome type 1 . Clin Genet 1999; 55: 50-54.

7 Van Tintelen P, Matthijs G, Braam W, Cassiman JJ, Duran M, Poll-The BT: Homozygosity for the F119L mutation in the $P M M 2$ gene in an adult with $\mathrm{CDG}$ syndrome type Ia. Eur J Hum Genet 1998; 6 (Suppl1): 62.

8 Stibler H, Jaeken J, Kristiansson B: Biochemical characteristics and diagnosis of the carbohydrate-deficient glycoprotein syndrome. Acta Paediatr Scand 1991; 375 (suppl): 21-31.
9 De Koning TJ, Dorland L, Van Diggelen OP et al: A novel disorder of N-glycosylation due to phosphomannose isomerase deficiency. Biochem Biophys Res Com 1998; 245: 38-42.

10 Van Schaftingen E, Jaeken J: Phosphomannomutase deficiency is a cause of carbohydrate-deficient glycoprotein syndrome type I. FEBS Lett 1995; 377: 318-320.

11 Studier FW, Moffatt BA: Use of bacteriophage T7 RNA polymerase to direct selective high-level expression of cloned genes. J Mol Biol 1986; 189: 113-130.

12 Higuchi R: Recombinant PCR. In Innis MA, Gelfand DH, Sninsky JJ, White TJ (eds). PCR Protocols. A Guide to Methods and Applications. Academic Press: San Diego, 1988, pp 177-183.

13 Schollen E, Pardon E, Heykants L et al: Comparative analysis of the phosphomannomutase genes PMM1, $P M M 2$ and PMM2 $\psi$ the sequence variation in the processed pseudogene is a reflection of the mutations found in the functional gene. Hum Mol Genet 1998; 7: 157-164.

14 Pirard M, Achouri Y, Collet JF, Schollen E, Matthijs G, Van Schaftingen E: Kinetic properties and tissular distribution of mammalian phosphomannomutase isozymes. Biochem J 1999; 339: 201-207.

15 Kepes F, Schekman R: The yeast SEC53 gene encodes phosphomannomutase. J Biol Chem 1988; 263: 9155-9161. 